\title{
Synthesis of silver nanoparticles in DMSO solutions of starch: a comparative investigation of native and soluble starches
}

\author{
N. E. Kochkina ${ }^{1}$, O. A. Skobeleva ${ }^{2}$, Yu. V. Khokhlova ${ }^{1}$ \\ ${ }^{1}$ G. A. Krestov Institute of Solution Chemistry of the Russian Academy of Sciences, \\ Ivanovo, Russia \\ ${ }^{2}$ Research Center for Nonlinear Wave Mechanics and Technology \\ of the Russian Academy of Sciences, Moscow, Russia \\ nek@isc-ras.ru, hohjul@mail.ru
}

PACS 81.16.Be

DOI 10.17586/2220-8054-2015-6-3-405-411

\begin{abstract}
Silver nanoparticles (AgNPs) were synthesized in solutions of native and soluble starches in DMSO for the first time. The starches acted as reducing and stabilizing agents simultaneously. The kinetics of the process and its activation energy were determined by using UV-vis spectroscopy. The DMSO solution of soluble starch was characterized by better reductive activity than the native starch solution. The morphology and dispersion characteristics of AgNPs sols were evaluated from transmission electron microscopy (TEM). Sols, including spherical particles with mean diameter $\left(\mathrm{D}_{m}\right) 42.8 \mathrm{~nm}$ and metal rod-like particles, were obtained by using the native starch solution. Morphologically uniform sols of spherical AgNPs with $\mathrm{D}_{m}=37.2 \mathrm{~nm}$ were formed in the soluble starch solution. On the basis of zeta potential measurements, it was shown that the stability of a AgNPs dispersion in the soluble starch solution was higher in comparison to the native starch solution.
\end{abstract}

Keywords: Silver nanoparcicles synthesis, starch, DMSO, dispersion analysis, zeta potential.

Received: 16 December 2014

Revised: 3 February 2015

\section{Introduction}

AgNPs exhibit unique physical, chemical and biocidal properties that make them attractive for application in different engineering branches and medicine [1-6]. Chemical methods of AgNPs synthesis in aqueous solutions, using reductants such as sodium borohydride, quercetin, etc. are most frequently used [7-9]. Among the chemical techniques of AgNPs synthesis, it should be especially noted that ecologically safe biomineralization methods exist which are based on the use of saccharides (glucose, fructose, and so on) and polysaccharides (cellulose, cellulose ethers) as reducing agents [10-16]. Starch was also successfully utilized in the process of AgNPs generation as a reducing and stabilizing agent simultaneously [17-19]. The authors pointed out that starch solutions including AgNPs could have pharmaceutical and biomedical application, owing to combination of antibacterial properties of $\mathrm{Ag}$ and biocompatibility of starch. It should also be noted that all known methods of AgNPs synthesis with participation of starch were performed in an aqueous medium and based on the application of water soluble starch.

In the present study, we prepared silver nanoparticles by utilizing DMSO-based starch solutions as the reducing and stabilizing medium for the first time. DMSO is a solvent that is commonly used to achieve complete dissolution of starch. DMSO is inexpensive, 
biodegradable and is categorized as a class 3 solvent, the lowest toxicity class to humans and the environment [20,21]. DMSO can easily penetrate biological membranes and has anti-inflammatory, analgesic, cryoprotective, prophylactic and radioprotective properties, whereupon this solvent is widely used as component of medicines [22-24]. Thus, composite materials combining the properties of DMSO, AgNPs and starch could be used for the preparation of new medical drugs.

The objective of this paper is to evaluate the kinetics features of AgNPs synthesis realized in DMSO solutions of native corn and soluble starches. Also, the morphology, size, and stability of the resulting AgNPs were characterized.

\section{Experimental section}

\subsection{Materials}

Corn starch and soluble starch (starch indicator) were obtained from CHIMMED Company. The amylose content and reducing values of starches were determined according [25] and [26] respectively. The amylose content was 28 and $41 \%$, and reducing values were 0.03 and 0.63 respectively, for corn and soluble starches. $\mathrm{AgNO}_{3}$ and DMSO were of analytical grade and were purchased from Sigma-Aldrich.

\subsection{Synthesis of AgNPs}

In this method, $0.025 \mathrm{~g}$ starch sample was placed in a $50 \mathrm{ml}$ volumetric flask and dissolved in $20 \mathrm{ml}$ DMSO under stirring over three days. Then, $0.02123 \mathrm{~g}$ of $\mathrm{AgNO}_{3}$ was added to the starch solution, the mixture was stirred well and diluted with DMSO (total volume $50 \mathrm{ml}$ ). The final concentration of the solution was $0.5 \mathrm{~g} / 1$ and $2.5 \times 10^{-3} \mathrm{M}$ for the starch and the salt, respectively. The sample was maintained at specific temperatures (45, 50,55 or $65^{\circ} \mathrm{C}$ ) for two hours.

\subsection{UV-visible spectral analysis}

The absorption spectra of DMSO starch solutions (native or soluble) and $\mathrm{AgNO}_{3}$ mixtures were recorded on an Agilent $8453 \mathrm{UV}-\mathrm{Vis}$ spectrophotometer, from 300 to $600 \mathrm{~nm}$. A solution containing $0.5 \mathrm{~g} / \mathrm{l}$ starch in DMSO was used as the blank.

The rate $(\gamma)$ of AgNPs formation was estimated from spectrophotometric data according next expression: $\gamma=\mathrm{A}_{\tau} / \mathrm{A}_{0}$, where $\mathrm{A}_{\tau}, \mathrm{A}_{0}$ - absorbance of the sample in the resonance band maximum at time $\tau$ and 0 min respectively.

\subsection{Transmission electron microscopy}

The morphology and dispersion of AgNPs prepared were investigated by transmission electron microscopy (EMV-100L instrument, operating voltage $50 \mathrm{kV}$ ) on the next day after synthesis. Before sample preparation for TEM analysis, the complement of the reduction of $\mathrm{Ag}$ ions to AgNPs was tested. For this purpose, $\mathrm{NaCl}$ was added in DMSO starch solutions containing AgNPs. There was no white precipitate and/ or turbidity of $\mathrm{AgCl}$ in the samples, thereby giving evidence for the complete reduction of $\mathrm{Ag}^{+}$ions to $\mathrm{Ag}^{0}$. Samples were deposited onto carbon-filmed grids, air-dried and examined under the microscope. Digital microscope photos were taken and analyzed using the software Matlab 7.0.

\subsection{Zeta-potential analysis}

The zeta potential of AgNPs samples was evaluated by Zetasizer Nano ZS analyzer (Malvern Instruments Ltd). The samples were equilibrated before measurements at $25^{\circ} \mathrm{C}$ for 300 seconds. Tests of each AgNPs sols under study were repeated 5 times. 


\section{Results and discussion}

The formation of AgNPs in solution is qualitatively manifested as a change of the sample coloration from colorless to yellow or brown, because of the interaction between metal particles' surface electrons with light (plasmon resonance). The absorption spectrum of AgNPs sol have characteristic surface plasmon band (SPB) and this allows the identification of cluster formation from metal atoms spectrophotometrically.

At room temperature $23^{\circ} \mathrm{C}$, there was no formation of $\mathrm{Ag}$ particles in all solution systems under study, which was due to the extremely low rate of $\mathrm{Ag}$ ion reduction under these conditions. Spectrophotometric registration of the metallic particle synthesis was possible at temperatures above $45^{\circ} \mathrm{C}$. Fig. 1 illustrates the change in the UV/Vis spectra of the $\mathrm{DMSO} /$ starch and $\mathrm{AgNO}_{3}$ mixtures observed at $65^{\circ} \mathrm{C}$ over different periods of time after sample preparation. It can be seen that the SPB with a maximum at $418 \mathrm{~nm}$ appeared in the spectra of the samples at 20 minutes after synthesis initiation. The spectral patterns were qualitatively the same for the both starch solutions. Variation of the reaction temperature over the $45-65^{\circ} \mathrm{C}$ range was not accompanied by any shift of the surface plasmon band (SPB). Observed data were in good agreement with the results of studies of the UV/Vis spectra of the water starch solutions containing AgNPs [16-18]. Therefore, it can be concluded that the spectral changes of the systems under study here were related to the synthesis of AgNPs from the $\mathrm{Ag}$ ions with participation of DMSO/starch solutions.

It is known, that saccharides may act as a reducing agent through its aldehyde group, which is able to be oxidized [11, 12, 27]. In case of starch, a polysaccharide, the reducing power is also believed to be the action of aldehyde terminals mainly [18]. Soluble starch, containing more reductive aldehyde termini than the native one, likely provides higher rate of AgNPs synthesis. To verify this assumption, process kinetics were estimated from spectrophotometric data. As shown in Fig. 2, the temperature dependence of the reaction rate $(\gamma)$ in both starch solutions was approximated by a straight line in the $\ln \gamma$ vs. $1 / \mathrm{T}$ coordinates and described by the Arrhenius equation [28]. The slopes of these lines gave activation energies of 64.9 and $54.2 \mathrm{~kJ} / \mathrm{mol}$ for the native and soluble starches respectively. It should also be noted that the solvent DMSO can also participate in the reduction of the silver cations [29]. Meanwhile, input of DMSO in the process rate evidently was equal for the both systems. So, these data demonstrate higher synthesis rate of AgNPs in the soluble starch system.

An important characteristic of AgNPs which affects to a large extent their application properties is the size of the particles. In order to investigate the morphology and size of AgNPs under study, transmission electron microscopy (TEM) was applied. The results for the samples generated at $65^{\circ} \mathrm{C}$ are presented on Fig. 3. The soluble starch solution appears to promote the formation of spherical AgNPs with a mean diameter $\left(\mathrm{D}_{m}\right)$ of $37.2 \mathrm{~nm}$ and a standard deviation $(\sigma)$ of $10.7 \mathrm{~nm}$. Application of native starch solution leads to the formation of AgNPs with two different geometric shapes, namely spherical particles, characterized by $\mathrm{D}_{m}=42.8 \mathrm{~nm}$ and $\sigma=19.8 \mathrm{~nm}$ and rodlike particles which had diameter ranging from 62 to $104 \mathrm{~nm}$ and length ranging from 375 to $1917 \mathrm{~nm}$.

These data demonstrate the fact that higher nanoparticles sizes and a polydispersity of metal sols were produced in the native starch medium. It can be supposed that one of the reasons that this phenomenon arises is the various macromolecular compositions of these starch samples. The main components of starch are two different homopolymers of dglucose: the mainly linear amylose and the heavily branched amylopectin. As it is known that only sufficiently long linear macromolecules can form protective shields around synthesized 


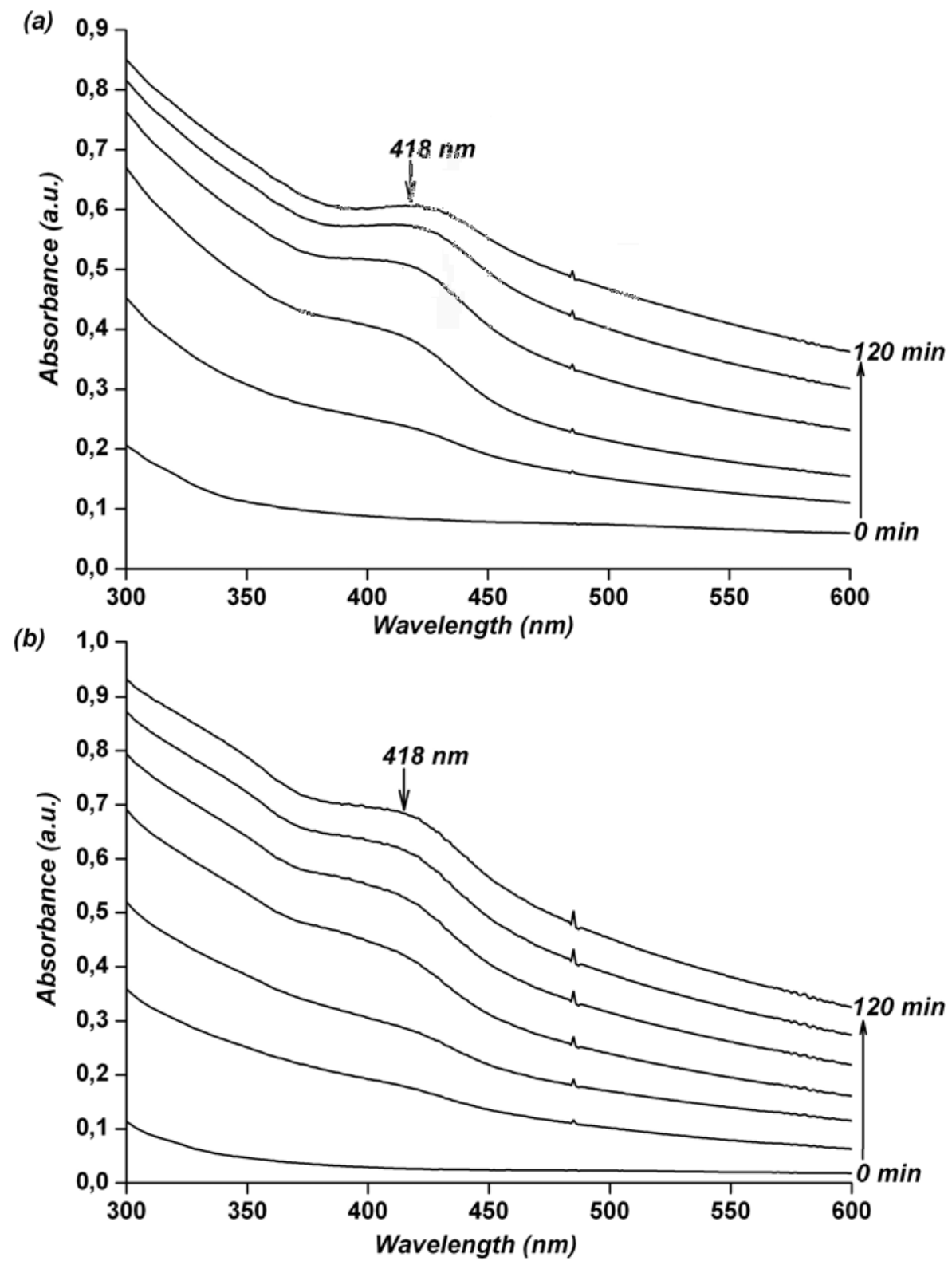

FIG. 1. UV-vis absorption spectra of the mixtures containing native (a) or soluble (b) starch solution in DMSO and $\mathrm{AgNO}_{3}$ within different time intervals after mixture's preparation. Reaction conditions: $[$ starch $]=0.5 \mathrm{~g} / \mathrm{l},\left[\mathrm{AgNO}_{3}\right]$ $=2.5 \times 10^{-3} \mathrm{M} / \mathrm{l}$, temperature $=65^{\circ} \mathrm{C}$

nanoparticles [30]. Amylopectin branched macromolecules with short side chains are not able to protect growing metal particles effectively. Thus, amylose chains are much more suitable for stabilizing the formation process than amylopectin. Therefore, soluble starch containing more amylose chains than the native starch provides formation of more morphologically uniform Ag nanoparticles with a narrower size distribution.

An essential factor for practical usage of colloidal systems is their stability, which can be evaluated by the measurement of the zeta potential of the dispersed particles. It is believed that systems including nanoparticles with zeta potential values greater than +25 


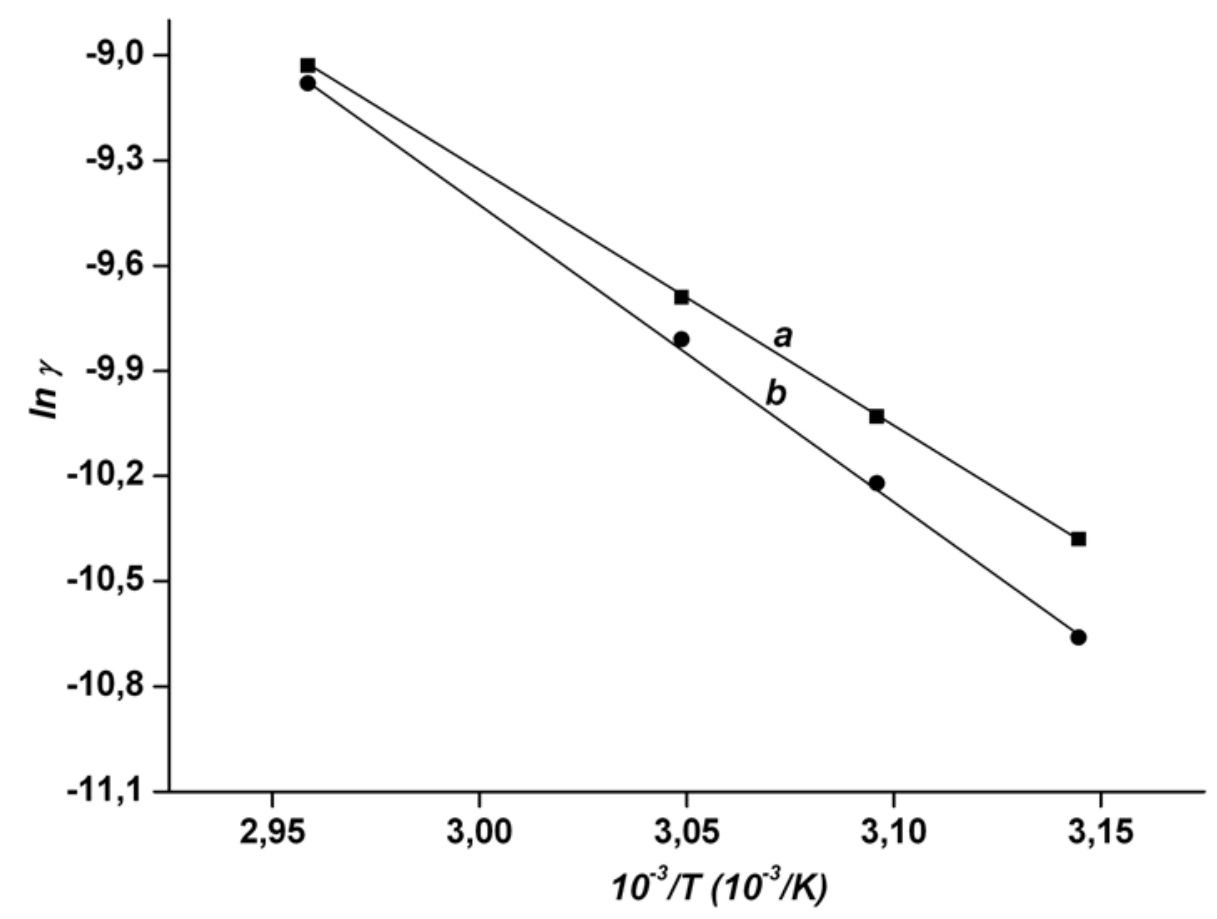

Fig. 2. Temperature dependence of the rate of AgNPs formation in the native (a) and soluble (b) starch solutions

$\mathrm{mV}$ or less than $-25 \mathrm{mV}$ typically have high degrees of stability. In our study, it was revealed that the zeta potentials of AgNPs synthesized in solutions of soluble and native starches were $+26 \pm 2 \mathrm{mV}$ and $+12 \pm 3 \mathrm{mV}$, respectively. The temperature of synthesis did not affect the values of AgNPs zeta potential. So these results demonstrated higher stability for AgNPs produced from DMSO solutions of soluble starch.

\section{Conclusions}

DMSO solutions of native and soluble starches were utilized for AgNPs synthesis. The kinetic features of the AgNPs formation in these two starch solutions were monitored by UV-vis spectroscopy. The activation energy for the reduction of Ag ions was less in case of soluble starch solution. According to the TEM micrograph measurements, rodlike and spherical particles were synthesized in the native starch solution. Soluble starch provided formation of $\mathrm{Ag}$ nanospheres which were characterized by smaller sizes and a narrower size distribution. The stability of AgNPs sol was better in case of those fabricated using the soluble starch solution.

\section{Acknowledgments}

The authors thank the center for joint use of scientific equipment "The upper Volga region center of physico-chemical research" for the spectrophotometric analysis and zeta potential measurements. 

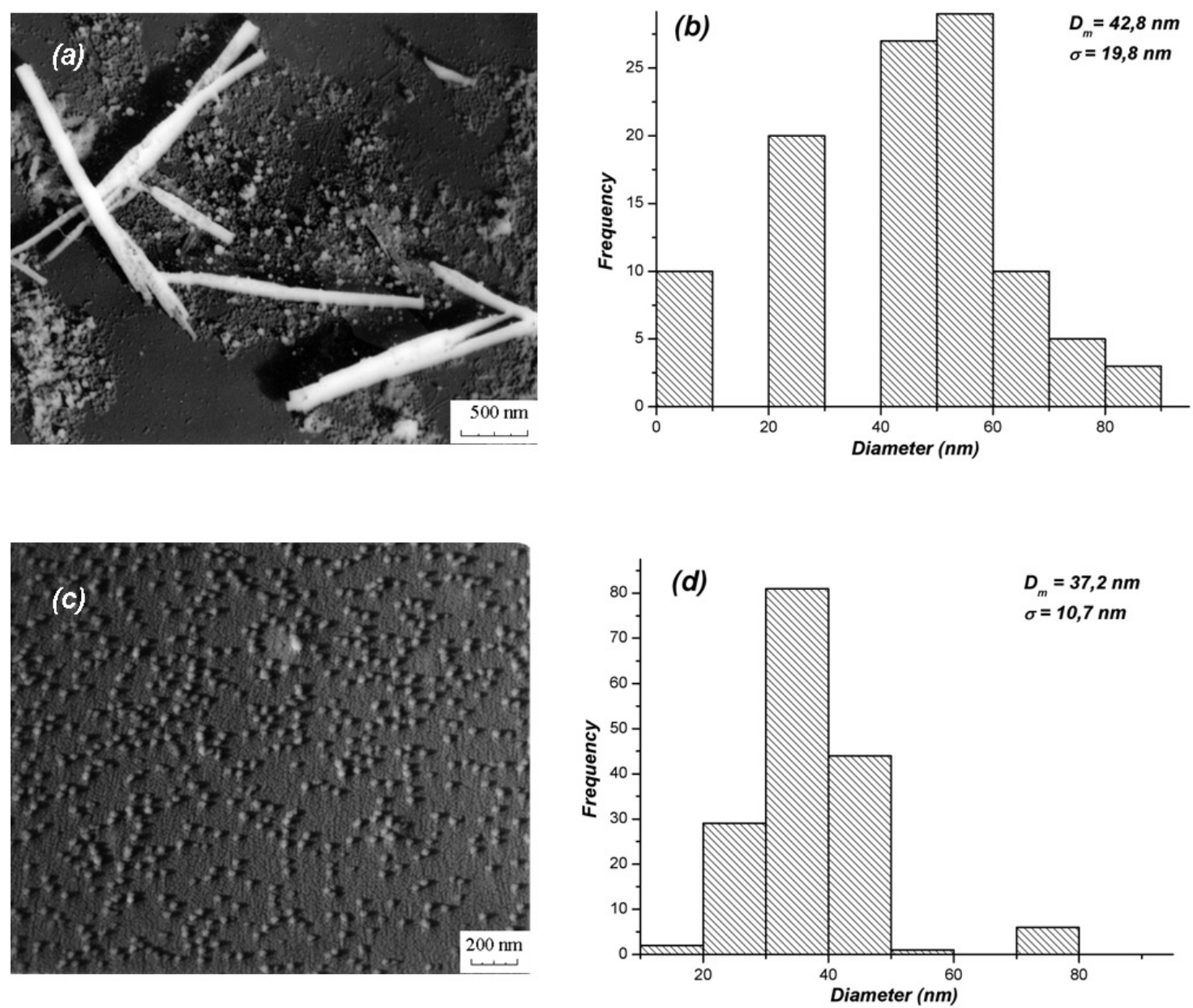

FIG. 3. TEM images (a, c) and particle size distribution histograms (b, d) of AgNPs prepared in the native (a, b) and soluble (c, d) starch solutions, synthesis temperature $=65^{\circ} \mathrm{C}$

\section{References}

[1] Henglein A. Small-particle research: physicochemical properties of extremely small colloidal metal and semiconductor particles. Chem. Rev., 1989, 89(8), P. 1861-1873.

[2] Welles A.E. (ed) Silver nanoparticles: properties, characterization and applications. Nova Science Publishers, StateplaceNew York, 2010, 397 p.

[3] Krutyakov Yu.A., Kudrinskiy A.A., Olenin A.Yu., Lisichkin G.V. Synthesis and properties of silver nanoparticles: advances and prospects. Russ. Chem. Rev., 2008, 77(3), P. 233-257.

[4] Silver S., Phung T., Silver G. Silver as biocides in burn and wound dressings and bacterial resistance to silver compounds. J. Ind. Microbiol. Biotechnol., 2006, 33(7), P. 627-634.

[5] Sarycheva A.S., Parshina E.Yu., Baydjumanov A.A., Semenova A.A., Goodilin E.A., Maximov G.V. Influence of silver hydrosols on structural integrity of erythrocytes. Nanosystems: Physics, Chemistry, Mathematics, 2013, 4(1), P. 66-71.

[6] Moushumy N., Alameh K., Rajendran V.,Yong Tak Lee. Silver-nanoparticle-based etch mask control for subwavelength structure development. Nanosystems: Physics, Chemistry, Mathematics, 2013, 4(3), P. 387-394.

[7] Caswell K.K., Bender C.B., Murphy C.J. Seedless, surfactantless wet chemical synthesis of silver nanowires. Nano Letters, 2003, 3(5), P. 667-669.

[8] Bois L., Chassagneux F., Battie Y., Bessueille F. Chemical growth and photochromism of silver nanoparticles into a mesoporous titania template. Langmuir, 2010, 26(2), P. 1199-1206. 
[9] Egorova E.M., Revina A.A. Synthesis of metallic nanoparticles in reverse micelles in the presence of quercetin. Colloids Surf. A, 2000, 168(1), P. 87-96.

[10] Venediktov E.A., Ganiev R.F., Padokhin V.A. Mechanism of formation of silver nanoparticle ensembles in an aqueous solution of glucose. Dokl. Chem., 2012, 442(5), P. 34-36.

[11] Hebeish A.A., El-Rafie M.H., Abdel-Mohdy F., Abdel-Halim E.A., et al. Carboxymethyl cellulose for green synthesis and stabilization of silver nanoparticles. Carbohydr. Polym., 2010, 82(3), P. 933-941.

[12] Abdel-Halima E.S., Salem S.S. Utilization of hydroxypropyl cellulose for green and efficient synthesis of silver nanoparticles. Carbohydr. Polym., 2011, 86(4), P. 1615-1622.

[13] Vysotsky V.V., Uryupina O.Ya., Roldughin V.I., Plachev Yu.A. Formation of silver nanoparticles in aqueous carboxymethyl cellulose solutions and the evolution of their sizes. Colloid J., 2009, 71(2), P. $156-162$.

[14] Raveendran P., Fu J., Wallen S. Completely "green" synthesis and stabilization of metal nanoparticles. J. Am. Chem. Soc., 2003, 125(46), P. 13940-13941.

[15] Gao X., Wei L., Yan H., Xu B. Green synthesis and characteristic of core-shell structure silver/starch nanoparticles. Materials Letters, 2011, 65(19-20), P. 2963-2965.

[16] Vasileva P., Donkova B., SnplaceKaradjova SnI., Dushkin C. Synthesis of starch-stabilized silver nanoparticles and their application as a surface plasmon resonance-based sensor of hydrogen peroxide. Colloid Surfaces A, 2011, 382(1-3), P. 203-210.

[17] Ghaseminezhada S.M., Hamedib S., Shojaosadati placecountry-regionS.A. Green synthesis of silver nanoparticles by a novel method: Comparative study of their properties. Carbohydr. Polym., 2012, 89(2), P. 467-472.

[18] Vigneshwaran N., Nachane R.P., Balasubramanya R.H., Varadarajan P.V. A novel one-pot 'green' synthesis of stable silver nanoparticles using soluble starch. Carbohydr. Res., 2006, 341(12), 20122008.

[19] Venediktov E.A., Ganiev R.F., Padokhin V.A. Preparation and stabilization of silver nanoparticles in liquid water soluble starch matrix. Dokl. Chem., 2012, 431(1), P. 34-36.

[20] ICH Topic Q3C (R4) - Impurities: Guideline for Residual Solvents. European Medicines Agency. Step 5, CityplaceLondon, 2011, 26 p.

[21] Vignes R. Dimethyl sulfoxide (DMSO) - A "New" clean, unique, superior solvent. American chemical society. Annual meeting. Washington, August 20-24, 2000.

[22] Santos N.C., Figueira-Coelho J., Martins-Silva J., Saldanha C. Multidisciplinary utilization of dimethyl sulfoxide: pharmacological, cellular, and molecular aspects. Biochem Pharmacol., 2003. 65(7), P. 10351041.

[23] Jacob S.W., de la Torre J.C.la Torre J.C. Pharmacology of dimethyl sulfoxide in cardiac and CNS damage. Pharmacological Reports, 2009, 61(2), P. 225-235.

[24] Young V.L., Boswell C.B., Centeno R.F., Watson M.E. DMSO: Applications in plastic surgery. Aesthet. Plast. Surg., 2005, 25(2), P. 201-209.

[25] Hoover R., Ratnayake W.S. Determination of total amylose content of starch. Current protocols in food analytical chemistry, 2001, E. 2.3.

[26] Whistler R.L., Wolfrom M.L. (eds.) Methods in Carbohydrate Chemistry, Vol. 1, Academic Press, StateplaceNew York, 1962, P. 380-394.

[27] Uryupina O.Ya., Vysotskii V.V., Matveev V.V., Gusel'nikova A.V., et al. Production of gold nanoparticles in aqueous solutions of cellulose derivatives. Colloid J., 2011, 73(4), P. 551-556.

[28] Moelwyn Hughes E.A. The chemical statics and kinetics of solutions. Academic Press, New York, $530 \mathrm{pp}$.

[29] Kuznetsova N.P., Ermakova T.G., Pozdnyakov A.S., Emel'yanov A.I., Prozorova G.F. Synthesis and characterization of silver polymer nanocomposites of 1-vinyl-1,2,4-triazole with acrylonitrile. Russ. Chem. Bull., 2013, 62(11), P. 2509-2513.

[30] Papisov, I.M., Litmanovich A.A. On recognition phenomena in polymer-minute particle interactions and pseudo-matrix processes. Colloids Surf. A., 1999, 151(3), P. 399-408. 